\title{
Ubiquitous antigen-specific T regulatory type 1 cells variably suppress hepatic and extrahepatic autoimmunity
}

\author{
Channakeshava Sokke Umeshappa, 'Jacques Mbongue, ${ }^{1}$ Santiswarup Singha, ${ }^{1}$ Saswat Mohapatra, ' Jun Yamanouchi,, Justin A. Lee, \\ Roopa Hebbandi Nanjundappa, ${ }^{1}$ Kun Shao, ${ }^{1}$ Urs Christen, ${ }^{2}$ Yang Yang, ${ }^{1,3}$ Kristofor K. Ellestad, ${ }^{1}$ and Pere Santamaria ${ }^{1,4}$ \\ IJulia McFarlane Diabetes Research Centre (JMDRC) and Department of Microbiology, Immunology and Infectious Diseases, Snyder Institute for Chronic Diseases and Hotchkiss Brain Institute, Cumming \\ School of Medicine, University of Calgary, Alberta, Canada. ${ }^{2}$ Pharmazentrum Frankfurt, Klinikum der Coethe Universität Frankfurt, Frankfurt, Germany. ${ }^{3}$ Department of Biochemistry and Molecular Biology, \\ Cumming School of Medicine, University of Calgary, Alberta, Canada. ${ }^{4}$ nstitut D'Investigacions Biomèdiques August Pi i Sunyer, Barcelona, Spain.
}

Peptide MHC class II-based (pMHClI-based) nanomedicines trigger the formation of multicellular regulatory networks by reprogramming autoantigen-experienced $\mathrm{CD}^{+} \mathrm{T}$ cells into autoimmune disease-suppressing $\mathrm{T}$ regulatory type 1 (TR1) cells. We have shown that pMHCII-based nanomedicines displaying liver autoimmune disease-relevant yet ubiquitously expressed antigens can blunt various liver autoimmune disorders in a non-disease-specific manner without suppressing local or systemic immunity against infectious agents or cancer. Here, we show that such ubiquitous autoantigen-specific $\mathbf{T}$ cells are also awakened by extrahepatic tissue damage and that the corresponding TR1 progeny can suppress experimental autoimmune encephalomyelitis (EAE) and pancreatic $\beta$ cell autoreactivity. In mice having EAE, nanomedicines displaying either ubiquitous or CNS-specific epitopes triggered the formation and expansion of cognate TR1 cells and their recruitment to the CNS-draining lymph nodes, sparing their liver-draining counterparts. Surprisingly, in mice having both liver autoimmunity and EAE, liver inflammation sequestered these ubiquitous or even CNS-specific TR1 cells away from the CNS, abrogating their antiencephalitogenic activity. In these mice, only the ubiquitous antigen-specific TR1 cells suppressed liver autoimmunity. Thus, the scope of antigen spreading in autoimmune disorders is larger than previously anticipated, involving specificities expected to be silenced by mechanisms of tolerance; the regulatory activity, but not the retention of autoreactive TR1 cells, requires local autoantigen expression.

\section{Introduction}

A growing body of evidence has established the feasibility of using antigen-specific approaches for the treatment of autoimmunity (1). We have shown that nanoparticles (NPs) coated with disease-relevant peptide MHC (pMHC) molecules (2) can resolve inflammation in various organ-specific autoimmune disease models in a disease-specific manner without impairing normal immunity (3-5). In all these models, pMHC class II-NP (pMHCII-NP) therapy functions by reprogramming cognate antigen-experienced $\mathrm{CD} 4^{+} \mathrm{T}$ cells into FoxP3-CD25- $\mathrm{T}$ regulatory type 1-like (TR1-like) cells, followed by systemic expansion. When these cells encounter costimulation-competent autoantigen-loaded antigen-presenting cells (APCs) in the target organ and proximal lymphoid tissues, they produce regulatory cytokines, including IL-10, TGF- $\beta$, and IL-21, leading to comprehensive inhibition of autoreactive $\mathrm{T}$ cell activation and recruitment and disease reversal.

NOD.c3c4 mice are resistant to T1D but develop a form of autoimmune biliary disease that resembles human primary biliary cholangitis (PBC) (6). Like human PBC, PBC in NOD.c3c4 mice

Conflict of interest: PS is scientific founder of Parvus Therapeutics.

Copyright: (5) 2020, American Society for Clinical Investigation.

Submitted: May 29, 2019; Accepted: January 3, 2020; Published: March 3, 2020.

Reference information: J Clin Invest. 2020;130(4):1823-1829.

https://doi.org/10.1172/JCl130670. is associated with spontaneous $\mathrm{T}$ and $\mathrm{B}$ cell responses against the mitochondrial pyruvate dehydrogenase (PDC) complex (7). Treatment of NOD.c3c4 mice with NPs displaying $\mathrm{IA}^{\mathrm{g} 7}$ presenting mPDC-E2 epitopes blunted the progression of, and reversed overt, PBC (5). In contrast, treatment with NPs coated with the pancreatic $\beta$ cell-specific $\mathrm{BDC}_{2.5 \mathrm{mi}} / \mathrm{IA}^{\mathrm{g} 7} \mathrm{pMHC}$ triggered neither TR1 cell expansion nor disease reversal. This outcome was consistent with the fact that pMHCII-NPs exclusively operate on autoantigen-experienced $\mathrm{CD}^{+}{ }^{+} \mathrm{T}$ cells (4).

Since PDC is an autoantigen expressed in virtually all cell types, our results raised the question of whether PBC-relevant nanomedicines were disease specific. This was addressed by investigating their pharmacodynamic activity in models of primary sclerosing cholangitis (PSC) and autoimmune hepatitis (AIH). We reasoned that bile duct or hepatocyte damage in PBC and PSC or AIH, respectively, would trigger the release of not only the PBC-relevant autoantigen PDC, but also the AIH-relevant autoantigens cytochrome P450 (CYP2D6) and formimidoyltransferase cyclodeaminase (FTCD), leading to the priming of autoreactive $\mathrm{CD}^{+} \mathrm{T}$ cells capable of responding to the corresponding pMHC-NPs. This was indeed the case (5). Remarkably, these therapeutic effects were dissociated from impairment of normal immunity (5).

The current study was initiated to investigate the following: (a) whether spontaneous or induced extrahepatic cell death can trigger the activation of ubiquitous antigen-specific $\mathrm{CD} 4^{+} \mathrm{T}$ 

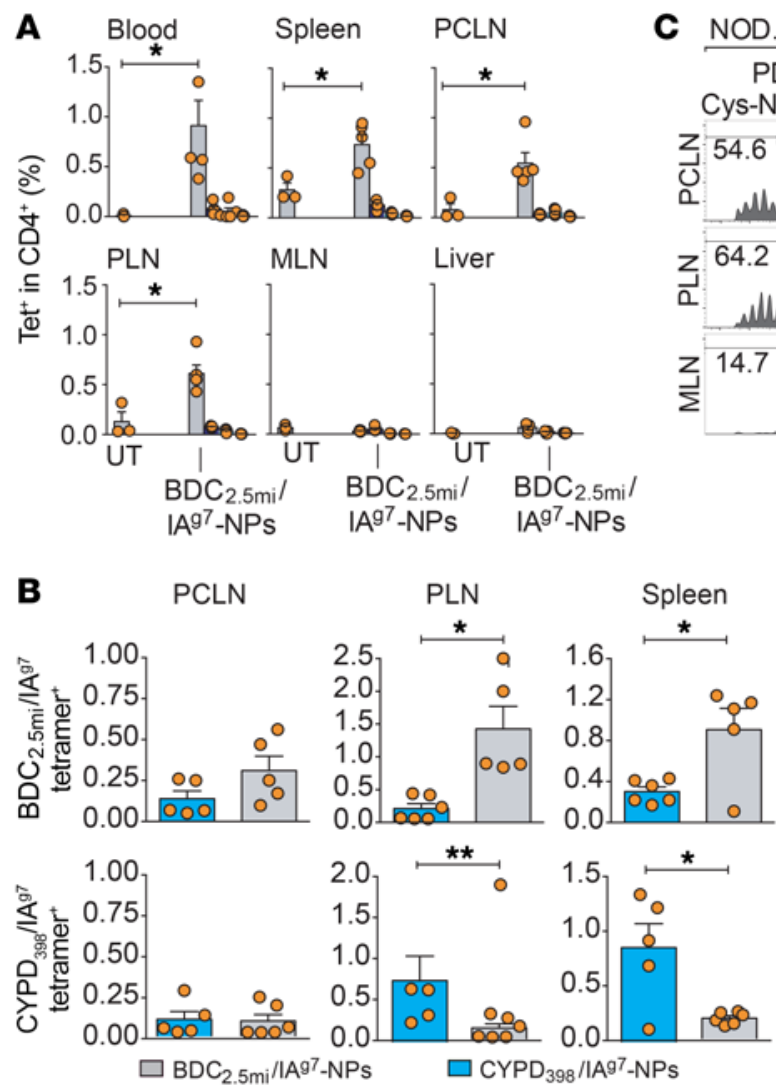

$/$ AA $^{97}-N P s$
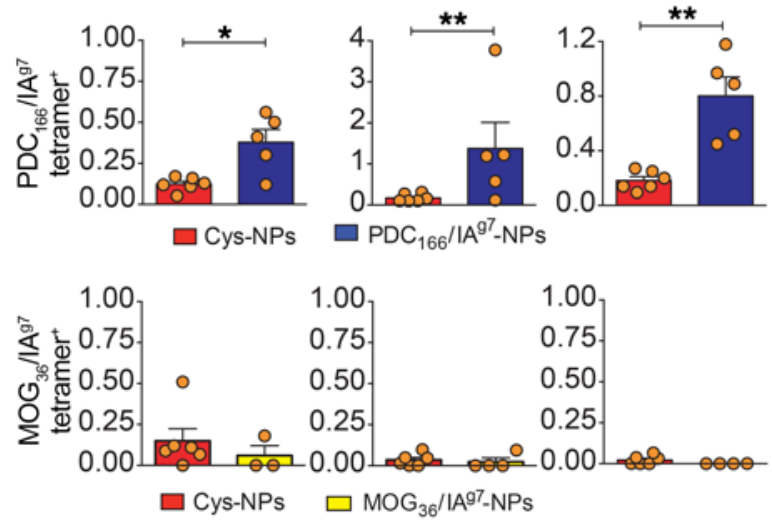

cells and thus render these cells responsive to the reprograming properties of pMHC-based nanomedicines and (b) whether the pharmacodynamic and therapeutic effects of these nanomedicines, displaying ubiquitous antigenic epitopes, are liver specific or also able to suppress extrahepatic autoimmunity as compared with pMHC-based nanomedicines displaying tissue-specific or irrelevant antigens.

\section{Results and Discussion}

Treatment of 10-week-old NOD mice with PDC-E2 ${ }_{166-181} / \mathrm{IA}^{\mathrm{g} 7}-$ NPs (PBC relevant) and $\mathrm{CYPD}_{398-412} / \mathrm{IA}^{\mathrm{g} 7}-\mathrm{NPs}$ (AIH relevant) did not trigger the expansion of cognate TR1-like $\mathrm{CD} 4^{+} \mathrm{T}$ cells relative to endogenous $\mathrm{BDC}_{2.5 \mathrm{mi}} / \mathrm{IA}^{\mathrm{g} 7}$-specific $\mathrm{T}$ cells from untreated mice in blood or other organs, including liver (Supplemental Figure 1, A and B; supplemental material available online with this article; https://doi.org/10.1172/JCI130670DS1). As expect-
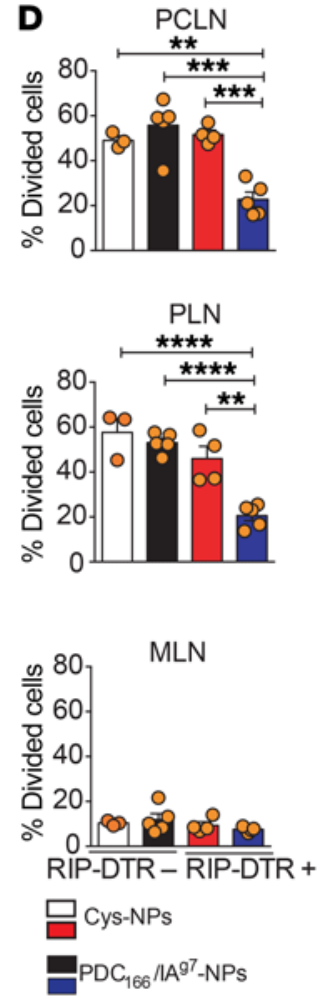

Figure 1. Autoantigen shedding renders autoreactive $\mathrm{T}$ cells responsive to PMHCII-NP displaying epitopes from ubiquitous autoantigens. (A) Percentages of tetramer+CD4+ cells in NOD mice upon treatment with Cys-NPs ( $n=$ 3) or $\mathrm{BDC}_{2.5 \mathrm{mi}} / / \mathrm{A}^{\mathrm{g} 7}-\mathrm{NPs}(n=5)$. (B) Percentages of tetramer ${ }^{+} \mathrm{CD}^{+}{ }^{+}$cells in DT-treated NOD.RIPhDTR mice following pMHCII-NP therapy. Data correspond to 6 Cys-NP-, $5 \mathrm{BDC}_{2.5 \mathrm{mi}} / I \mathrm{~A}^{\mathrm{g} 7}-\mathrm{NP}-, 5$ $\mathrm{PDC}_{166-181} / / \mathrm{A}^{\mathrm{g} 7}-\mathrm{NP}-, 5 \mathrm{CYPD}_{398-412} / \mathrm{AA}^{\mathrm{g} 7}-\mathrm{NP}-$ and 3 to $4 \mathrm{MOG}_{36-50} / / \mathrm{A}^{\mathrm{g}} \mathrm{-NP}-\mathrm{Nreated}$ mice (2 experiments). (C) Proliferation of CFSE ${ }^{+} 8.3-\mathrm{CD}^{+}$ T cells from 8.3-NOD.G6pc2 ${ }^{-1-}$. $\mathrm{Tra}^{-1-}$ donors in the PCLN, PLN, and MLN of DT-treated NOD.RIP-hDTR or NOD mice that received $\mathrm{PDC}_{166-181} / \mathrm{IA}^{\mathrm{g} 7}-\mathrm{NPs}$ or Cys-NPs. Data correspond to 3 Cys-NP-treated NOD.RIP-hDTR (1 experiment); $5 \mathrm{PDC}_{166-181} / \mathrm{IA}^{\mathrm{g} 7}$-NP-treated NOD (1 experiment); 4 Cys-NP-treated NOD.RIPhDTR ( 2 experiments); and $4 \mathrm{PDC}_{166-181} / / \mathrm{A}^{\mathrm{g} 7}-\mathrm{NP}-$ treated NOD.RIP-hDTR mice (2 experiments). (D) Average percentages of divided cells in the mice from $\mathbf{C}$. Data correspond to the mean \pm SEM. $P$ values were calculated using Mann-Whitney $U$ (A and B) or 1-way ANOVA with Tukey's post hoc correction (D). ${ }^{*} P<0.05$; ${ }^{* *} P<0.01$; ${ }^{* *} P<0.001$; and ${ }^{* * * *} P<0.0001$. ed, $\mathrm{BDC}_{2.5 \mathrm{mi}} / \mathrm{IA}^{\mathrm{g} 7}$-NPs triggered the formation of cognate TR1like $\mathrm{CD} 4^{+} \mathrm{T}$ cells and their accumulation in the LNs draining both liver and pancreas (portal/celiac LNs [PCLNs] and pancreatic LNs [PLNs]), but not in nondraining LNs (mesenteric LNs [MLNs]) or in the liver (Figure 1A and Supplemental Figure 1, C and $\mathrm{D})$. This suggested that spontaneous $\beta$ cell killing in prediabetic NOD mice does not trigger the formation of PDC-E2 or CYPD2D6 autoantigen-experienced T cells capable of responding to pMHCII-NPs. We entertained 3 alternative possibilities to explain these results: (a) these antigens are released from cholangiocytes and hepatocytes (in liver autoimmunity), but not from dying $\beta$ cells (in type 1 diabetes [T1D]); (b) the antigens are released from $\beta$ cells, but NOD mice do not export cognate autoreactive $\mathrm{T}$ cells for these antigens; or (c) the antigens are released and the mice harbor cognate $\mathrm{T}$ cells, but the antigens are released in insufficient amounts. 
A
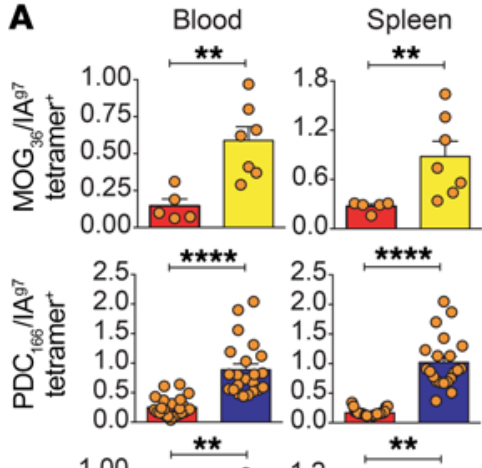

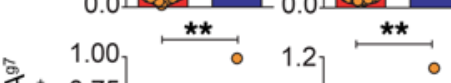

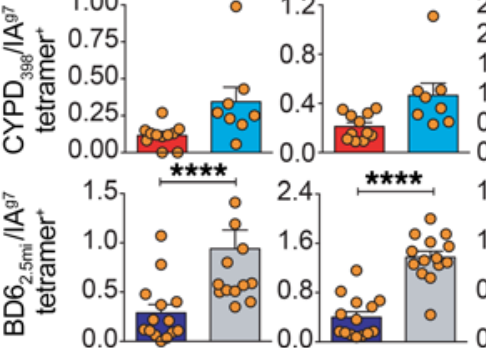

C

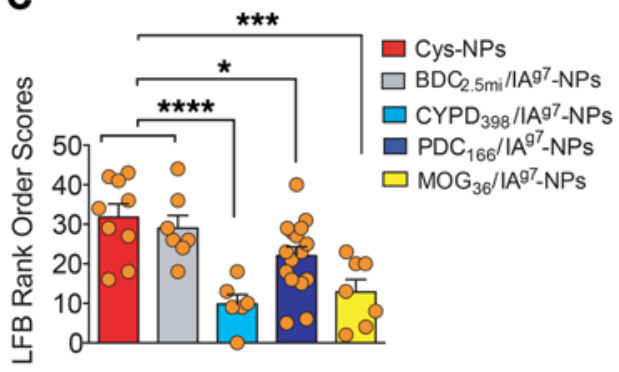

CLN PCLN Liver
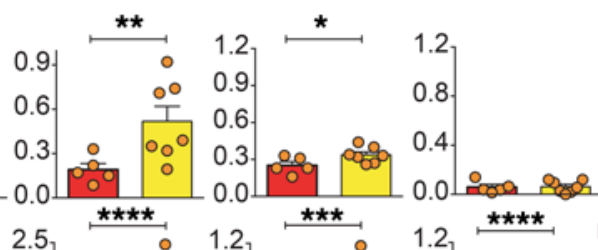

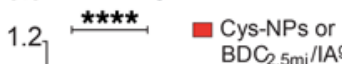

- Cys-NPs $\square \mathrm{MOG}_{36} / \mathrm{IA}^{97}-\mathrm{NPS}$

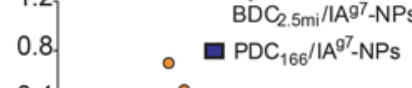

$0.4 \%$
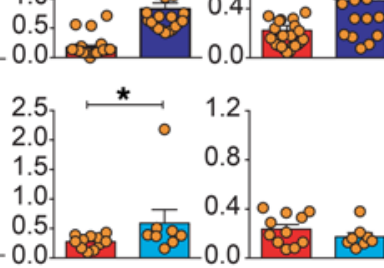

$\left.\begin{array}{l}1.2 \\ 0.8 \\ 0.4 .1 .200 \\ 0.0\end{array}\right]$

1.2
0.8

0.8 .

$0.4 \div \quad:$

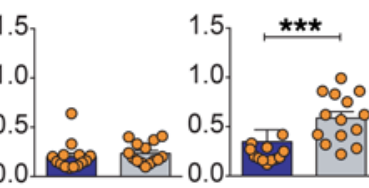

1.5
1.0
0.5
0.0
D

- Cys-NPs $\because \mathrm{MOG}_{38} / \mathrm{IA}$-NPs - $\mathrm{PDC}_{94} / \mathrm{IA}^{\mathrm{b}}-\mathrm{NPS} \quad \mathrm{CYPD}_{353} / \mathrm{IA}^{\mathrm{b}}-\mathrm{NPS}$

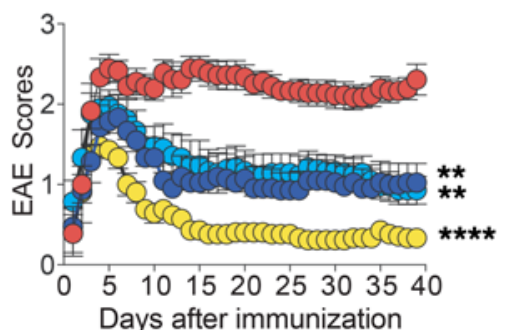

Days after immunization
B ${ }^{2.0} 7$ Treatment start

崖
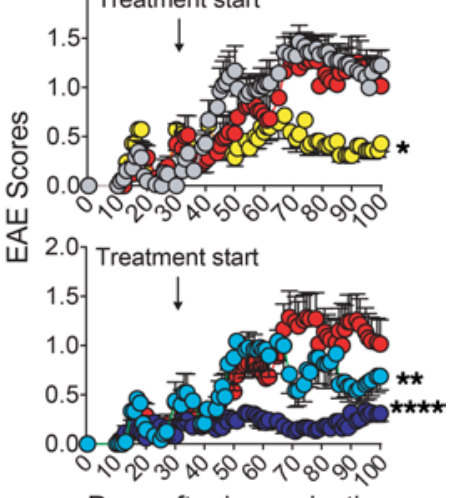

Days after immunization

Cys-NPs

- $\mathrm{PDC}_{166} / \mathrm{IA}^{97}$-NPs

(.) $M O G_{36} / I^{97}-N P s$

- $\mathrm{BDC}_{2.5 \mathrm{mi}} / \mathrm{AA}^{97}$-NPs

$\mathrm{CYPD}_{398} / \mathrm{IA}^{97}$-NPs

\section{E}

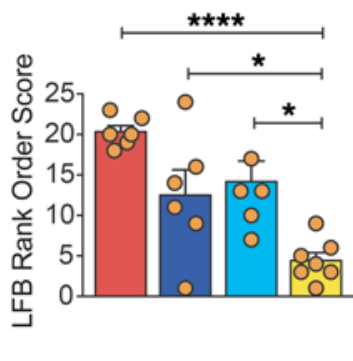

$\square$ Cys-NPs

$\square \mathrm{PDC}_{94} / \mathrm{IA}^{\mathrm{b}}-\mathrm{NPs}$

$\square \mathrm{CYPD}_{353} / \mathrm{IA}^{\mathrm{b}-N P S}$

$\square \mathrm{pMOG}_{38} / \mathrm{IA}^{\mathrm{b}}-\mathrm{NPS}$

Figure 2. TR1 cells recognizing epitopes from ubiquitous autoantigens are recruited to the CNS and blunt both relapsing-remitting and chronic-progressive EAE. (A) Percentages of tetramer ${ }^{+} \mathrm{CD} 4^{+}$cells in pMHCII-NP-treated NOD mice with EAE. Data (from left to right) correspond to 5 Cys-NP- and 7 $\mathrm{MOG}_{36-50} / / \mathrm{A}^{\mathrm{g} 7}-\mathrm{NP}$-treated mice; 11 Cys-NP- plus $14 \mathrm{BDC}_{2.5 \mathrm{mmi}} / \mathrm{IA}^{\mathrm{g} 7}-\mathrm{NP}$ - and $22 \mathrm{PDC}_{166-181} / \mathrm{IA}^{\mathrm{g} 7}-\mathrm{NP}$-treated mice (2 experiments); 11 Cys-NP- and $8 \mathrm{CYPD}_{398-412} /$ $\mathrm{IA}^{\mathrm{g} 7}$-NP-treated mice (2 experiments); and $14 \mathrm{PDC}_{166-181} / \mathrm{IA}^{\mathrm{g} 7}-\mathrm{NP}$ - and $14 \mathrm{BDC}_{2.5 \mathrm{mi}} / \mathrm{IA}^{\mathrm{g} 7}-\mathrm{NP}$-treated mice. (B) EAE scores in NOD mice treated with Cys-NPs $(n=11), \mathrm{BDC}_{2.5 \mathrm{mi}} / \mathrm{IA}^{\mathrm{g7}}-\mathrm{NPs}(n=14)$, or $\mathrm{MOC}_{36-50} / \mathrm{IA}^{\mathrm{g} 7}-\mathrm{NPs}(n=8), \mathrm{PDC}_{166-181} / \mathrm{IA}^{\mathrm{g}}-\mathrm{NPs}(n=22)$ or $\mathrm{CYPD}_{398-412} / \mathrm{I}^{\mathrm{g}}-\mathrm{NPs}(n=8)$ from 2 experiments. (C) Average blinded rank order Luxol fast blue (LFB) scores for the mice shown in B. Data (mean \pm SEM) correspond to $n=9,7,6,16$ and 7 mice/NP type, from left to right. (D) EAE scores in C57BL/6 mice. Data correspond to 6 Cys-NP-, $7 \mathrm{MOG}_{38-49} / \mathrm{IA}^{\mathrm{b}-N P}-, \mathrm{PDC}_{94-108} / \mathrm{IA}^{\mathrm{b}}-\mathrm{NP}-$ and $7 \mathrm{CYPD}_{353-367} / \mathrm{IA}^{\mathrm{b}}-\mathrm{NP}-\mathrm{treated}$ mice. (E) Average (mean \pm SEM) blinded rank order LFB scores for mice shown in D. Data correspond to $n=6,6,6$, and $7 / p M H C-N P$ type, from left to right. $P$ values were calculated using 2-way ANOVA (B and $\mathbf{D})$, Mann-Whitney $U$ test $(\mathbf{A})$, or 1-way ANOVA with Tukey's post hoc correction $(\mathbf{C}$ and $\mathbf{E})$. ${ }^{*} P<0.05 ;{ }^{* *} P<0.01$; ${ }^{* * *} P<0.001 ;$ and ${ }^{* * * *} P<0.0001$

To distinguish among these possibilities, we expressed an $\mathrm{X}$ chromosome-linked rat-insulin promoter-driven human diphtheria toxin (DT) receptor (hDTR) transgene in NOD mice and administered DT to kill about $50 \%$ of $\beta$ cells (due to X chromosome inactivation, only $50 \%$ express the hDTR) (8). We then gave DT-treated mice PDC-E2 ${ }_{166-181} / \mathrm{IA}^{\mathrm{g} 7}-\mathrm{NPs}, \quad \mathrm{CYPD}_{398-412} /$ $\mathrm{IA}^{\mathrm{g} 7}$-NPs (PBC/AIH relevant), $\mathrm{BDC}_{2.5 \mathrm{mi}} / \mathrm{IA}^{\mathrm{g} 7}$-NPs (T1D-specific), $\mathrm{MOG}_{36-50} / \mathrm{IA}^{\mathrm{g} 7}$-NPs (experimental autoimmune encephalomyelitis specific [EAE-specific]) or Cys-coated NPs (control). As expected, $\mathrm{MOG}_{36-50} / \mathrm{IA}^{\mathrm{g} 7}$-NPs did not expand cognate tetramer ${ }^{+}$ $\mathrm{CD}^{+} \mathrm{T}$ cells (Figure 1B). In contrast, both PDC-E2 ${ }_{166-181} / \mathrm{IA}^{\mathrm{g} 7}-$ and $\mathrm{CYPD}_{398-412} / \mathrm{IA}^{\mathrm{g} 7}-\mathrm{NPs}$ triggered the formation and accumulation of cognate $\mathrm{T}$ cells expressing the TR1 markers LAG3, $\mathrm{LAP}$, and CD49b in the spleen and PLNs, to an extent similar to that seen for $\mathrm{BDC}_{2.5 \mathrm{mi}} / \mathrm{IA}^{\mathrm{g} 7}$ tetramer ${ }^{+} \mathrm{T}$ cells in $\mathrm{BDC}_{2.5 \mathrm{mi}} / \mathrm{IA}^{\mathrm{g} 7}-\mathrm{NP}-$ treated animals (Figure 1B and Supplemental Figure 1, E and F). Furthermore, whereas the PCLNs and PLNs of NOD.RIP-hDTR mice treated with DT plus Cys-NPs supported the proliferation of exogenous CFSE-labeled IGRP ${ }_{206-214}$-specific CD8 ${ }^{+} \mathrm{T}$ cells (upon recognition of $\beta$ cell-derived IGRP $206-214$ draining into these LNs), neither the PCLNs nor PLNs of NOD.RIP-hDTR mice treated with DT plus PDC-E2166-181/IA ${ }^{\mathrm{g} 7}$-NPs could do so (Figure 1, C and $\mathrm{D}$ ). These observations support the third scenario described earlier and demonstrate that the PDC-E2 ${ }_{166-181} / \mathrm{IA}^{\mathrm{g} 7}$-NP-induced TR1 cells that accumulate in the PCLNs/PLNs suppress the activation of noncognate $\beta$ cell-autoreactive T cells by local APCs (loaded with $\beta$ cell-derived PDC-E2 and IGRP). In addition, these findings corroborate that PMHC-NP-induced TR1 cell formation requires autoantigen-experienced $\mathrm{T}$ cells (absent in nonDT-treated mice) (Figure 1, A-D and Supplemental Figure 1, A 

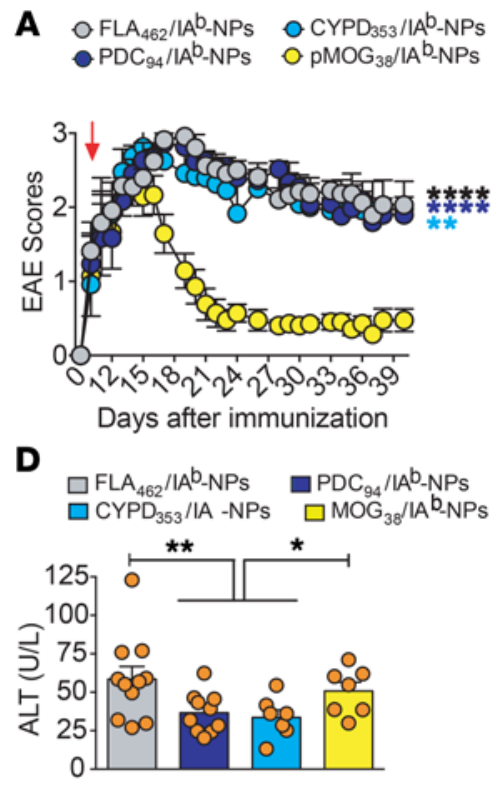
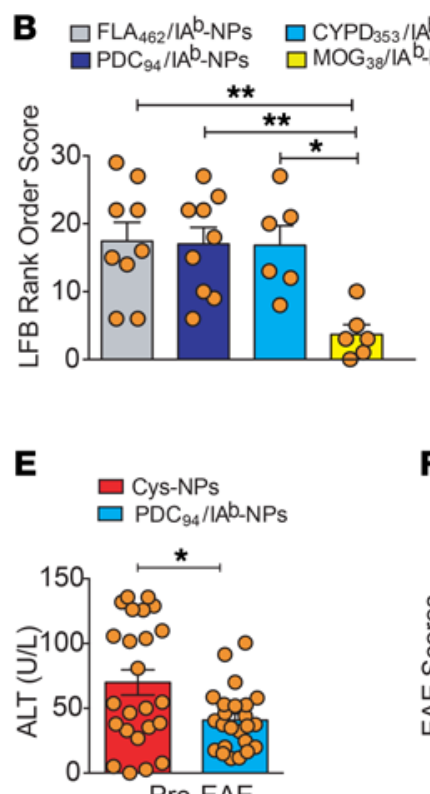

Pre-EAE
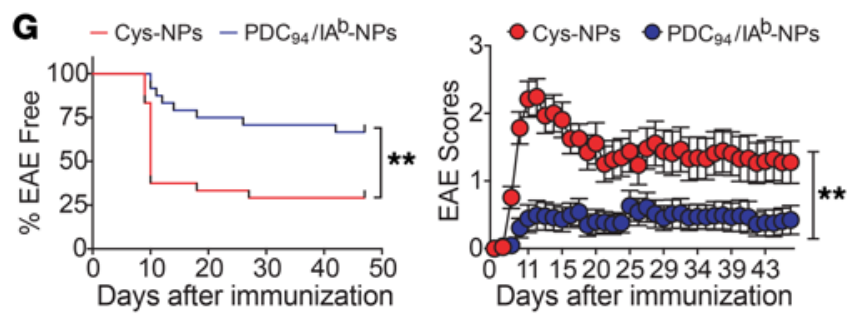

Figure 3. In mice with both EAE and AIH, ubiquitous antigen-specific TR1 cells are selectively recruited to the liver. (A) EAE scores are shown: 11 $\mathrm{Fla}_{462-472} / \mathrm{IA}^{\mathrm{b}}-\mathrm{NP}, 7 \mathrm{MOC}_{38-49} / \mathrm{IA}^{\mathrm{b}}-\mathrm{NP}, 10 \mathrm{PDC}_{94-108} / \mathrm{IA}^{\mathrm{b}}-\mathrm{NP}$, and $7 \mathrm{CYPD}_{353-367} / \mathrm{IA}^{\mathrm{b}}-\mathrm{NP}$. (B) Average rank order LFB scores. Data correspond to $n=9$, 9, 6, and 6 mice (left to right). (C) Average histopathological scores from the mice shown in $\mathbf{A}$. Data correspond to $n=11,10$, 7, and 7 mice (left to right). (D) Serum ALT levels: $n=11,10,7$, and 7 mice (left to right). (E) Serum ALT levels in B6 mice with AIH receiving Cys-NPs ( $n=24)$ or PDC-E2 ${ }_{94-108} / / A^{\text {b }}$ -

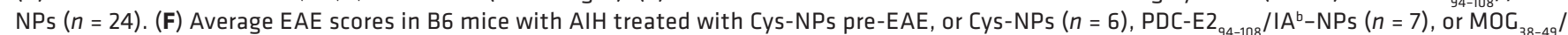
$\mathrm{IA}^{\mathrm{b}}$-NPs $(n=7)$ after EAE induction. (G) EAE incidence (left) and average EAE scores (right) of B6 mice having AIH that received Cys-NPs ( $\left.n=24\right)$ or PDC-E2 ${ }_{94-108} /$ IA $^{\mathrm{b}}-\mathrm{NPs}(n=24)$ before EAE. (H) Therapeutic effect of PDC-E2 ${ }_{94-108} / \mathrm{IA}^{\mathrm{b}}-\mathrm{NPs}(n=5)$ versus Cys-NPs $(n=3)$ in PDC-E2 ${ }_{94-108} / \mathrm{IA}^{\mathrm{b}}-\mathrm{NP}-$ treated B6 mice with $\mathrm{AlH}$, after EAE induction. Data correspond to mean \pm SEM values. $P$ values were calculated using 2 -way ANOVA (A, F, G [right panel], and $\mathbf{H})$, Kaplan-Meier survival (G [left panel]), Mann-Whitney $U$ test (E), or 1-way ANOVA with Tukey's post hoc correction (B, C, and D). ${ }^{*} P<0.05$; ${ }^{*} P<0.01$; and ${ }^{*}{ }^{*}{ }^{*} P<0.0001$.

and B). Furthermore, they indicate that NOD mice harbor T cells targeting ubiquitously expressed antigens and that the priming of such cells requires antigen shedding.

We next asked whether the therapeutic effects of these ubiquitous antigen-based nanomedicines were liver specific. We compared the ability of PDC-E2 $2_{166-181} / \mathrm{IA}^{\mathrm{g} 7}-\mathrm{NPs}$ and $\mathrm{CYPD}_{398-412} /$ $\mathrm{IA}^{\mathrm{g} 7}-\mathrm{NPs}$ vs. $\mathrm{BDC}_{2.5 \mathrm{mi}} / \mathrm{IA}^{\mathrm{g} 7}-\mathrm{NPs}$ and $\mathrm{MOG}_{36-50} / \mathrm{IA}^{\mathrm{g} 7}-\mathrm{NPs}$ to blunt $\mathrm{MOG}_{36-55}$-induced EAE in NOD mice; disease kinetics/severity were similar to those reported earlier (9). $\mathrm{BDC}_{2.5 \mathrm{mi}} / \mathrm{IA}^{\mathrm{g} 7}-\mathrm{NPs}$ expanded cognate TR1-like T cells as in non-EAE-affected NOD mice (4), but these cells were absent from the CNS-draining cervical LNs (CLNs) and liver (Figure 2A and Supplemental Figure 1, G-I) and had no antiencephalitogenic activity (Figure 2, B and $\mathrm{C}$; and Supplemental Figure 1J). Notably, both CYPD $\mathrm{D}_{398-412} / \mathrm{IA}^{\mathrm{g7}-}$ NPs and PDC-E $2_{166-181} / I^{\mathrm{g} 7}-\mathrm{NPs}$ also triggered TR1 cell expansion (Figure 2A and Supplemental Figure 1, G-I), but unlike $\mathrm{BDC}_{2.5 \mathrm{mi}} / \mathrm{IA}^{\mathrm{g} 7}-\mathrm{NPs}$, suppressed EAE (Figure 2, B and C; and Supplemental Figure 1J) in association with accumulation of cog- nate TR1-like cells in the CLNs (Figure 2A). These effects were also seen in C57BL/6 mice with EAE that received PDC-E2 ${ }_{94-108} /$ $\mathrm{IA}^{\mathrm{b}}-\mathrm{NPs}$ or $\mathrm{CYPD}_{353-367} / \mathrm{IA}^{\mathrm{b}}$-NPs (Figure 2, D and E; Supplemental Figure 2, A-C; and Supplemental Figure 3A). Thus, upon oligodendrocyte damage, both PDC-E2 and CYPD2D6 are delivered to proximal APCs for autoreactive $\mathrm{CD}^{+} \mathrm{T}$ cell priming, enabling TR1 cell generation by pMHC-NPs, recruitment to the CLNs, and suppression of EAE.

We next asked whether accumulation of pMHCII-NP-induced TR1 cells is driven by inflammation in a non-antigen-specific manner. We tracked $\mathrm{MOG}_{38-49} / \mathrm{IA}^{\mathrm{b}-}$, PDC-E2 $2_{94-108} / \mathrm{IA}^{\mathrm{b}-}$, and $\mathrm{CYPD}_{353-36 /} / \mathrm{IA}^{\mathrm{b}}$-specific TR1 cells arising in pMHCII-NP-treated B6 mice having both AIH and EAE (Supplemental Figure $4 \mathrm{~A}) . \mathrm{MOG}_{38-49} / \mathrm{IA}^{\mathrm{b}}-\mathrm{NPs}$ triggered expansion and accumulation of cognate TR1-like $\mathrm{CD} 4^{+} \mathrm{T}$ cells in blood, spleen, and CLNs, but not in the liver, PCLNs, or MLNs (Supplemental Figure 5, A-C). Mice treated with $\mathrm{Fla}_{462-472} / \mathrm{IA}^{\mathrm{b}}-\mathrm{NPs}$, displaying a colitis-relevant gut microbial epitope, lacked both $\mathrm{MOG}_{38-49} / \mathrm{IA}^{\mathrm{b}}-$ and $\mathrm{Fla}_{462-472} /$ 

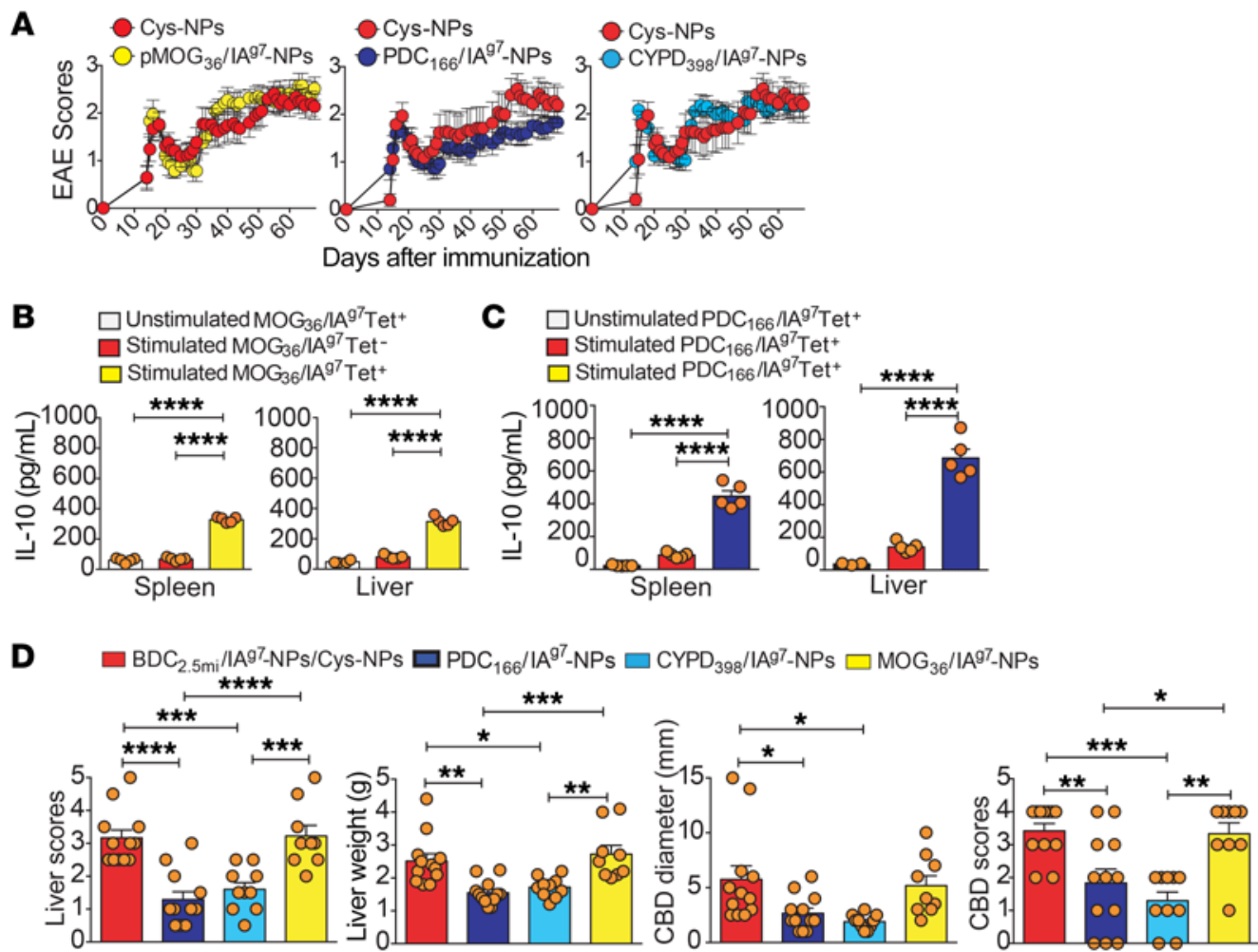

\section{E $\square \mathrm{BDC}_{2,5 \mathrm{mi}} / / \mathrm{AA}^{97}$-NPs/Cys-NPs $\square \mathrm{PDC}_{166} / / \mathrm{A}^{97}$-NPs
$\square \mathrm{CYPD}_{398} / \mathrm{A}^{97}$-NPs $\quad \square \mathrm{MOG}_{36} / \mathrm{IA}^{97}$-NPs}
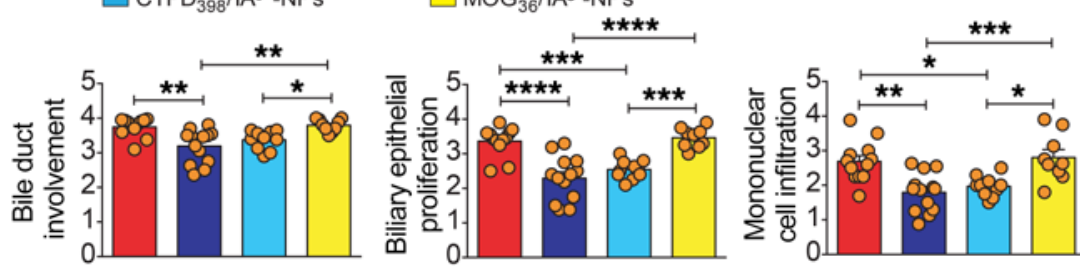

Figure 4. In mice having both EAE and PBC, CNS autoantigen-specific TR1 cells are sequestered in the liver. (A) EAE scores in pMHCII-NP-treated NOD.c3c4 mice having both PBC and EAE. Data (from left to right) correspond to $n=16$ mice receiving control $\left(C y s-N P\right.$ and $\left.B D C_{2.5 \mathrm{mi}} / I A^{\mathrm{g} 7}-\mathrm{NP}\right)$ or $n=$ 11 mice receiving $\mathrm{MOG}_{36-50} / \mathrm{IA}^{\mathrm{g} 7}$-NPs (left); $n=11$ mice receiving Cys-NP or $n=14$ mice receiving $\mathrm{PDC}_{166-191} / \mathrm{IA}^{\mathrm{g} 7}-\mathrm{NPs}$ (middle); and $n=11$ mice receiving Cys-NPs or $n=12$ mice receiving $\mathrm{CYPD}_{398-412} / \mathrm{IA}^{\mathrm{g} 7}$-NPs (right). (B and C) IL-10 secretion by FACS-sorted spleen- and liver-derived $\mathrm{MOG}_{36-50} / \mathrm{IA}^{\mathrm{g} 7}-(\mathbf{B})$ and $\mathrm{PDC}_{166-181} / \mathrm{IA}^{\mathrm{g} 7}-(\mathrm{C})$ tetramer ${ }^{+}$and tetramer $\mathrm{CD}^{+} \mathrm{T}$ cells in response to stimulation with anti-CD3/CD28 mAb-coated beads or no stimulation. Data correspond to $n=4-5$ samples/group. (D) Liver scores, liver weight, CBD diameter, and CBD scores from the mice shown in $\mathbf{A}$. Data correspond to $n=$ $12,13,10$, and 9 mice/NP type. (E) Average PBC histopathological scores from the mice shown in $\mathbf{A}$. Data correspond to $n=12,12,10$, and 9 mice/NP type. Data are represented as mean \pm SEM. $P$ values were calculated using 2-way ANOVA (A) or 1-way ANOVA with Tukey's post hoc correction (B-E). ${ }^{*} P<0.05 ;{ }^{*} P<0.01 ;{ }^{* *} P<0.001 ;$ and ${ }^{* * *} P<0.0001$.

$\mathrm{IA}^{\mathrm{b}}$-specific $\mathrm{CD} 4^{+} \mathrm{T}$ cells (Supplemental Figure $5 \mathrm{~A}$ ). As a result, $\mathrm{MOG}_{38-49} / \mathrm{IA}^{\mathrm{b}}-\mathrm{NPs}$, but not $\mathrm{Fla}_{462-42} / \mathrm{IA}^{\mathrm{b}}-\mathrm{NPs}$, reversed EAE (Figure 3, A and B; and Supplemental Figure 3B) without suppressing AIH (Figure 3, C and D; and Supplemental Figure 5D).

Surprisingly, treatment with PDC-E2 $2_{94-108} / \mathrm{IA}^{\mathrm{b}}-$ or CYPD $_{353-367} /$

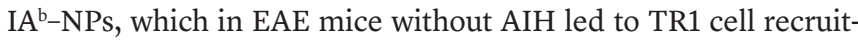
ment to the CLNs and EAE reversal (Figure 2, D and E; and Supplemental Figure 2A), resulted in the accumulation of these cells in the liver and liver-draining LNs but not in the CLNs (Supplemental Figure $5, \mathrm{~A}-\mathrm{C})$. As a result, $\mathrm{PDC}-\mathrm{E} 2_{94-108} / \mathrm{IA}^{\mathrm{b}}-\mathrm{NPs}$ and $\mathrm{CYPD}_{353-36} /$ IA $^{\mathrm{b}}$-NPs suppressed liver disease (Figure 3, C and D; and Supplemental Figure 5D), but not EAE (Figure 3, A and B). Additional experiments in a NOD model of AIH (Ad-hFTCD induced) confirmed that recruitment of such ubiquitous antigen-reactive TR1- like cells to the liver was antigen driven; treatment of these mice with $\mathrm{BDC}_{2.5 \mathrm{mi}} / \mathrm{IA}^{\mathrm{g}}{ }^{7} \mathrm{NPs}$ triggered cognate $\mathrm{TR} 1$ cell recruitment to the PLNs and PCLNs, but not to the CLNs, MLNs, or liver (Supplemental Figure 5, E-G). Thus, accumulation of pMHC-NPinduced TR1 cells to sites of inflammation and draining lymphoid tissue and the ensuing therapeutic effects require local autoantigen expression. Whereas this is also true for TR1 cells recognizing ubiquitous antigens, liver inflammation sequesters these cells away from the CNS.

To further probe the role of active liver inflammation in the sequestration of ubiquitous antigen-specific TR1-like cells, we tracked their recruitment in mice in which EAE was induced upon treatment of AIH (Supplemental Figure 4B). As expected, PDC-E2 ${ }_{94-108} / \mathrm{IA}^{\mathrm{b}}$-NPs reduced serum ALT levels versus what was 
found in Cys-NP-treated controls (Figure 3E). We then immunized mice having treated or untreated $\mathrm{AIH}$ with $\mathrm{MOG}_{36-55}$ to induce EAE. Treatment of the mice that received Cys-NPs after AIH induction with PDC-E2 ${ }_{94-108} / \mathrm{IA}^{\mathrm{b}}-\mathrm{NPs}$ triggered the recruitment of PDC-E2 ${ }_{94-108} / \mathrm{IA}^{\mathrm{b}}$-reactive $\mathrm{CD}^{+} \mathrm{T}$ cells to the PCLNs and liver, but not to the CLNs (as compared with mice that continued to receive Cys-NPs after AIH-induction; Supplemental Figure $5 \mathrm{H}$ ), and failed to reverse EAE (Figure $3 \mathrm{~F}$ and Supplemental Figure 3C). In contrast, treatment with $\mathrm{MOG}_{38-49} / \mathrm{IA}^{\mathrm{b}-\mathrm{NPs}}$ triggered the recruitment of cognate $\mathrm{CD} 4^{+} \mathrm{T}$ cells to the CLNs, but not PCLNs or liver, and reversed EAE (Figure 3F, Supplemental Figure 3C, and Supplemental Figure 5H). Only treatment with PDC-E2 ${ }_{94-108} /$

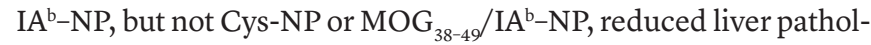
ogy (Supplemental Figure 5I). Thus, continued liver inflammation

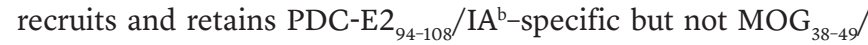
IA $^{\mathrm{b}}$-specific TR1 cells.

Remarkably, the mice that received PDC-E2 ${ }_{94-108} / \mathrm{IA}^{\mathrm{b}}-\mathrm{NPs}$ after AIH induction were resistant to EAE (Figure 3G and Supplemental Figure 3D). Furthermore, both the mice that did not develop EAE and those that received PDC-E2 ${ }_{94-108} / \mathrm{IA}^{\mathrm{b}-N P s}$ after developing EAE had larger accumulations of cognate $\mathrm{CD} 4^{+} \mathrm{T}$ cells in the CLNs (as well as PCLNs) than the mice that received Cys-NPs after EAE induction (Supplemental Figure 5H), consistent with the EAE resistance of the former and therapeutic responsiveness of the latter to PDC-E2 ${ }_{94-108} / \mathrm{IA}^{\mathrm{b}-\mathrm{NPs}}$ (Figure 3H, Supplemental Figure 3E, and Supplemental Figure 5J). Both types of mice had improved liver pathology versus those that received Cys-NP after EAE induction or Cys-NPs after AIH induction (Supplemental Figure 5I). Thus, resolution of liver inflammation releases PDC-E2 ${ }_{94-108} /$

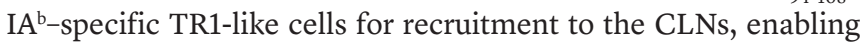
them to blunt EAE.

We next superimposed EAE onto the more aggressive, chronic form of liver autoimmunity that develops in NOD.c3c4 mice (Supplemental Figure 4C) (5). The severity of EAE in NOD.c3c4 mice was greater than in NOD mice, suggesting that liver inflammation does not nonspecifically sequester effector CNS-autoreactive $\mathrm{CD}^{+}{ }^{+} \mathrm{T}$ cells. We treated these mice with PDC-E2 ${ }_{166-181} / \mathrm{IA}^{\mathrm{g} 7}-\mathrm{NPs}$, $\mathrm{CYPD}_{398-412} / \mathrm{IA}^{\mathrm{g} 7}-\mathrm{NPs}, \mathrm{MOG}_{36-50} / \mathrm{IA}^{\mathrm{g} 7}-\mathrm{NPs}, \mathrm{BDC}_{2.5 \mathrm{mi}} / \mathrm{IA}^{\mathrm{g} 7}-\mathrm{NPs}$, or Cys-NPs. Surprisingly, none of these nanomedicines suppressed the progression of EAE (Figure 4A; Supplemental Figure 3F). The cognate TR1-like $\mathrm{CD} 4^{+} \mathrm{T}$ cells induced by these nanomedicines were present in the liver and PCLNs, but not in the CLNs or MLNs (Supplemental Figure 6, A-C). Both the splenic and liver-associated $\mathrm{MOG}_{36-50} / \mathrm{IA}^{\mathrm{g} 7}$ - and PDC-E2 ${ }_{166-181} / \mathrm{IA}^{\mathrm{g} 7}$-specific $\mathrm{CD}^{+} \mathrm{T}$ cells of these mice were TR1 like because they coexpressed LAG-3 and CD49b (Supplemental Figure 6, D and E) and produced IL-10 in response to TCR ligation ex vivo, unlike their unstimulated counterparts or stimulated tetramer- $\mathrm{CD}^{+} \mathrm{T}$ cells from the same mice (Figure 4, B and C).

Recruitment of the PDC-E2 ${ }_{166-181} / \mathrm{IA}^{\mathrm{g} 7}-$ and $\mathrm{CYPD}_{398-412} / \mathrm{IA}^{\mathrm{g} 7}-$ specific TR1-like CD4 ${ }^{+} \mathrm{T}$ cells to the liver and PCLNs was associated with improved liver pathology (Figure 4, D and E; and Supplemental Figure 6, F-H). In contrast, although $\mathrm{MOG}_{36-50} /$ $\mathrm{IA}^{\mathrm{g} 7}-\mathrm{NP}$-induced TR1 $\mathrm{CD} 4^{+} \mathrm{T}$ cells were recruited to the liver and PCLNs, they did not suppress liver disease, indicating that their immunoregulatory effects require local autoantigen expression (Figure 4, D and E; and Supplemental Figure 6, F-H). Thus, severe liver inflammation can efficiently retain antigen-specific TR1 cells nonspecifically. The larger size, hence higher antigenic load, of the liver versus the CNS coupled to the fenestrated liver vasculature may underlie the preferential recruitment of ubiquitous autoantigen-specific TR1 cells to this organ upon inflammation. Additionally, our data indicate that the degree of inflammation (i.e., PBC vs. AIH) and the magnitude of antigen shedding (i.e., spontaneous vs. DT induced) also play important roles. Interestingly, pMHCII-NP-induced TR1 cells upregulate CCR5 and CXCR3 and downregulate CCR7 mRNA as compared with conventional $\mathrm{CD}^{+} \mathrm{T}$ cells (our unpublished data). Since portal vessel-derived CCR5 ligands and liver sinusoid-derived CXCR3 ligands have been implicated in lymphocyte recruitment to the liver, and sinusoidal and lymphatic vessel-derived CCR7 ligands in lymphocyte egress from the liver (10), differential expression of these chemokine receptors may also play a role. Future studies will be needed to determine whether sequestration of ubiquitous autoantigen-specific TR1 cells in the liver will also occur in mice in which liver autoimmunity develops after extrahepatic autoimmunity or whether these phenomena are applicable to disease/organ pairs other than the liver/CNS axis.

Collectively, our results show that pMHCII-based nanomedicines displaying ubiquitous antigenic epitopes can blunt not only various liver autoimmune disorders, but also CNS-specific autoimmunity, albeit not as efficiently as nanomedicines displaying CNS-specific autoantigens. Importantly, our work exposes a biological phenomenon whereby autoreactive $\mathrm{T}$ cells against ubiquitous antigens (11) are awakened by antigen shedding from different cells/tissues, including hepatocytes, cholangiocytes, pancreatic $\beta$ cells, and oligodendrocytes, rendering them responsive to $\mathrm{pMHCII}$-based nanomedicines. This implies that central and peripheral mechanisms of tolerance for systemically expressed autoantigens such as PDC-E2 and CYPD2D6, including clonal deletion and functional inactivation, are not fully penetrant, even in nonautoimmune-prone genetic backgrounds. In turn, this implies that the scope of epitope- and antigen-spreading in autoimmune disorders is much larger than previously anticipated. Furthermore, our observations not only highlight the essential role for local autoantigen expression in the regulatory activity of antigen-specific TR1-like cells but also indicate that liver inflammation has the potential to nonspecifically draw $\mathrm{T}$ regulatory cells away from sites of cognate autoantigen expression and autoimmune inflammation (Supplemental Table 1).

\section{Methods}

The methods are described in the Supplemental Methods.

Study approval. These studies were approved by the animal care committee of the Cumming School of Medicine.

\section{Author contributions}

CSU produced the pMHCs and executed most of the experiments shown in Figures 1-4 and Supplemental Figures 1-6 with contributions from JM, SS, SM, JAL, JY, RHN, KS, UC, YY, and KKE and contributed to writing the manuscript with PS. PS designed the study, supervised and coordinated its execution, and wrote the manuscript with CSU. 


\section{Acknowledgments}

We thank H. Jamaleddine and A. Khadra for theoretical contributions to the concept of competitive autoimmunity; S. Thiessen, J. Erickson, G. Mendizabal and J. Fetsch, Y. Liu, L. Kennedy, and K. Poon for technical contributions; and the staff of the Nicole Perkins Microbial Communities Core. This work was funded by the Canadian Institutes of Health Research (CIHR), Diabetes Canada, the Multiple Sclerosis Society of Canada, the Crohn's and Colitis Foundation of Canada, and MINECO (RTI2018-093964-B-I00). CSU was supported by CIHR, Alberta-Innovates-Health-Solutions, and Banting-CIHR fellowships. The JMDRC was supported by Diabetes Canada.

Address correspondence to: Pere Santamaria, Department of Microbiology, Immunology and Infectious Diseases, Cumming School of Medicine, University of Calgary, Alberta, T2N 4N1 Canada. Phone: 403.220.8735; Email: psantama@ucalgary.ca.
1. Serra P, Santamaria P. Antigen-specific therapeutic approaches for autoimmunity. Nat Biotechnol. 2019;37(3):238-251.

2. Singha S, et al. Peptide-MHC-based nanomedicines for autoimmunity function as T-cell receptor microclustering devices. Nat Nanotechnol. 2017;12(7):701-710.

3. Tsai $S$, et al. Reversal of autoimmunity by boosting memory-like autoregulatory T cells. Immunity. 2010;32(4):568-580.

4. Clemente-Casares X, et al. Expanding antigen-specific regulatory networks to treat autoimmunity. Nature. 2016;530(7591):434-440.
5. Umeshappa CS, et al. Suppression of a broad spectrum of liver autoimmune pathologies by single peptide-MHC-based nanomedicines. Nat Commun. 2019;10(1):2150.

6. Irie J, et al. NOD.c3c4 congenic mice develop autoimmune biliary disease that serologically and pathogenetically models human primary biliary cirrhosis. J Exp Med. 2006;203(5):1209-1219.

7. Kita H, et al. Quantitative and functional analysis of PDC-E2-specific autoreactive cytotoxic T lymphocytes in primary biliary cirrhosis. J Clin Invest. 2002;109(9):1231-1240.
8. Thorel F, et al. Conversion of adult pancreatic alpha-cells to beta-cells after extreme beta-cell loss. Nature. 2010;464(7292):1149-1154.

9. Papenfuss TL, et al. Sex differences in experimental autoimmune encephalomyelitis in multiple murine strains. J Neuroimmunol. 2004;150(1-2):59-69.

10. Oo YH, Adams DH. The role of chemokines in the recruitment of lymphocytes to the liver. JAutoimmun. 2010;34(1):45-54.

11. Moon JJ, et al. Quantitative impact of thymic selection on Foxp3+ and Foxp3- subsets of self-peptide/ MHC class II-specific CD4+ T cells. Proc Natl Acad Sci US A. 2011;108(35):14602-14607. 